\title{
The WNT5A Agonist Foxy5 Reduces the Number of Colonic Cancer Stem Cells in a Xenograft Mouse Model of Human Colonic Cancer
}

\author{
JANINA OSMAN, KISHAN BELLAMKONDA ${ }^{\S}$, QING LIU, TOMMY ANDERSSON* and ANITA SJÖLANDER* \\ Cell and Experimental Pathology, Department of Translational Medicine, \\ Clinical Research Centre, Lund University, Malmö, Sweden
}

\begin{abstract}
Background: The wingless-type mammary tumour virus integration site 5A (WNT5A) agonist Foxy5 was shown in vitro to affect intracellular signalling implicated in the regulation of colonic cancer stem cells (CSCs). Materials and Methods: In order to study whether Foxy5 can modulate CSCs, either HT-29 or Caco-2 human colonic cancer cells, both lacking endogenous WNT5A expression, were inoculated subcutaneously into nude mice. Results: Foxy5 reduced the expression of the stem-cell marker aldehyde dehydrogenase and, interestingly, the specific colon CSC marker double cortin-like kinase 1. Foxy5 also reduced active $\beta$-catenin and the expression of its downstream target Achaete Scute complex homolog 2, a CSC-preserving transcription factor. Foxy5 also reduced cyclo-oxygenase 2 expression, responsible for the formation of the CSC-promoting prostaglandin $E_{2}\left(P G E_{2}\right)$, but increased that of 15-hydroxyprostaglandin dehydrogenase expression, a $P G E_{2}$-degrading enzyme. Accordingly, Foxy5 impairs both $\beta$-catenin and $P G E_{2}$ signalling, both of which have been implicated in promoting the niche of colonic CSCS. Conclusion: Our data suggest that Foxy5 can complement the traditional adjuvant chemotherapeutic treatment to which CSCs are resistant.
\end{abstract}

This article is freely accessible online.

*These Authors contributed equally to this study.

\$Present address: Division of Clinical Genetics, Lund University, Malmö, Sweden.

Correspondence to: Professor Anita Sjölander, Cell and Experimental Pathology, Department of Translational Medicine, Lund University, Clinical Research Centre, Malmö, Sweden. Tel: +46 40391168, e-mail: anita.sjolander@med.lu.se

Key Words: WNT5A, Foxy5 peptide, tumour cell signalling, cancer stem cells.
Colonic cancer is among the most common types of cancer in both women and men $(1,2)$. This form of cancer is routinely staged (I to IV) based on the size and extent of the primary tumour, the number of lymph nodes engaged by the disease and the absence or presence of distant metastases $(1,2)$. The choice of treatment of patients with colonic cancer depends on the stage of the tumour, but also on the age and well-being of the patient. The primary treatment of these patients is surgery, which can be complemented with chemotherapy (3, 4). A major problem despite such treatments is the risk of relapse of the disease that increases with stage.

An essential factor for relapse is the survival of tumourinitiating cells with 'stem-like' characteristics (5). These tumour-initiating cells are distinct from non-malignant stem cells and exhibit low proliferative rates, high self-renewing capacity, multi-potency, and the ability to differentiate into actively proliferating tumour cells $(5,6)$, thus explaining why they are also referred to as cancer stem cells (CSCs). Their resistance to chemotherapy further characterizes CSCs (5). A fundamental problem in cancer research is, therefore, how to reduce or eliminate CSCs. Colon CSCs are promoted by elevated intracellular $\beta$-catenin and prostaglandin $\mathrm{E}_{2}\left(\mathrm{PGE}_{2}\right)$ signalling (6). These two distinctly different signalling pathways are obvious targets in the search for a possible therapeutic approach aiming at reducing the number of CSCs to thereby delay or even prevent cancer relapse. Interesting in this context are the findings from in vitro experiments that wingless-type mammary tumour virus integration site $5 \mathrm{~A}$ (WNT5A) signalling simultaneously reduces both $\beta$-catenin and $\mathrm{PGE}_{2}$ signalling in colonic cancer cells (7).

WNT5A is a complex ligand belonging to the WNT family of proteins, consisting of 19 different ligands (8). These ligands are cysteine-rich proteins with 23-24 conserved cysteine residues, and require post-translational modifications, including covalent binding of lipids and glycosylation on asparagine residues, for proper secretion and signalling abilities (9). WNT ligands are traditionally characterized as either $\beta$-catenin (canonical) signalling ligands or non- $\beta$-catenin (non-canonical) 
signalling ligands. The WNT5A protein is the most intensely studied non- $\beta$-catenin signalling member of the WNT family (10). In human colonic cancer cells, WNT5A signalling has been shown to impair cell migration and invasion (11). These findings are in good agreement with the observations in human clinical tumour tissue that loss of WNT5A protein expression is associated with reduced patient survival (11). These results suggest that it would be beneficial for patients with colonic cancer that has reduced or lacks endogenous expression of WNT5A to be treated by reconstituting WNT5A signalling. The obvious approach would be to systemically administer recombinant WNT5A protein. However, this is not possible due to its size, complex structure and ability to bind to circulating proteins and cell structures.

In order to reconstitute WNT5A signalling in cancer cells that lack endogenous expression of WNT5A, we developed a hexapeptide derived from one of the solvent-exposed amino acid sequences of the WNT5A molecule; in addition, it was formylated on its N-terminal methionine (12). This hexapeptide, named Foxy5, is a proper WNT5A agonist in that it triggers numerous signalling events and functional responses as recombinant WNT5A, but in comparison with WNT5A, it is a much simpler molecule that can be administered systemically and still reach tumour tissue $(12,13)$. It also appears to have low toxicity, as noted in two successful clinical phase I trials (www.clinicaltrials.gov; NCT02020291 and NCT02655952). In in vitro experiments using human colonic cancer cells, the Foxy5 peptide, as well as recombinant WNT5A, impaired $\beta$-catenin signalling, reduced the expression of the $\mathrm{PGE}_{2}$-generating enzyme cyclo-oxygenase 2 (COX2) and increased the expression of the $\mathrm{PGE}_{2}$-degrading enzyme 15-hydroxyprostaglandin dehydrogenase (15-PGDH) (7), suggesting that Foxy-5 might influence the CSC niche. The interaction of CSCs with the tumour microenvironment is crucial for their ability to maintain their stemness properties. This is why regulation of CSCs is best studied in in vivo situations.

These findings prompted us to investigate, in the present study, whether the drug candidate Foxy5 could reduce the number of colonic CSCs in colonic cancer tissue from a mouse xenograft model using two different human colonic cancer cell lines.

\section{Materials and Methods}

Cell lines and chemicals. Both cancer cell lines were procured from the American Type Culture Collection (ATCC; Manassas, VA, USA). HT-29 colonic cancer cells (ATCC HTB-38 ${ }^{\mathrm{TM}}$ with a mutant carboxy-truncated adenomatous polyposis coli (APC) gene but a wild-type of the Kirsten rat sarcoma virus (KRAS) gene were grown in McCoy's 5A medium with glutamine. Caco-2 colonic cancer cells (ATCC HTB-37 ${ }^{\mathrm{TM}}$ with a mutated APC gene but a wild-type KRAS gene) were grown in Dulbecco's modified Eagle's medium (DMEM). Both media were supplemented with $10 \%$ foetal bovine serum and $100 \mu \mathrm{g} / \mathrm{ml}$ penicillin/streptomycin, and both cell types were maintained at $37^{\circ} \mathrm{C}$ in a humidified atmosphere containing $5 \%$ $\mathrm{CO}_{2}$. The cells were routinely screened for the absence of mycoplasma contamination until they were injected into the flanks of the mice (see below). Bachem (Bubendorf, Switzerland) manufactured the Foxy5 peptide that was then provided courtesy of WntResearch AB (Malmoe, Skane, Sweden).

Animal experiments. The female nude mice (BALB/c nu/nu; 5-6 weeks old) used in this study were purchased from Taconic Europe A/S (Ry, Denmark), and all animal experiments were approved by the Regional Ethics Committee for Animal Research at Lund University, Sweden (M163-15). Colonic cancer xenografts were induced by subcutaneous injection of $2.5 \times 10^{6}$ low-passage human HT-29 or Caco- 2 cells into both flanks of the mice ( $\mathrm{n}=10$ mice each for HT-29 and Caco-2 cells) (14). Tumour development was monitored by palpation. Once palpable tumours were detected (day 7), the mice were randomly divided into two groups for each cell line and treated with either vehicle alone $(\mathrm{NaCl})$ or Foxy5. The mice received a total of nine intraperitoneal injections of either vehicle or Foxy5 (40 $\mu \mathrm{g}$ per dose, $\sim 2 \mu \mathrm{g} / \mathrm{g}$ ) every second day from day 7 to day 23 . The dose of Foxy 5 was selected on the basis of published data $(13,15)$. The volume of HT-29- and Caco-2-derived tumours in animals treated with either vehicle alone or with Foxy5 were measured. The tumour diameters ( $\mathrm{d} 1$ and $\mathrm{d} 2$ ) were determined with a calliper the day before the animals were sacrificed, and the volume of each tumour was then calculated according to the formula, $V=(\pi / 6)\left(d_{1} \times d_{2}\right)^{3 / 2}(16)$.

After 24 days, all mice were sacrificed, and their tumours were removed. One tumour from each animal was frozen at $-80^{\circ} \mathrm{C}$, and the other tumour was fixed in $4 \%$ paraformaldehyde at $4^{\circ} \mathrm{C}$ for 48 hours, washed with water, dehydrated with increasing concentrations of ethanol, immersed in xylene and finally embedded in paraffin. The embedded tumour tissues were then investigated by immunohistochemical (IHC) staining.

IHC staining and intensity scoring. The paraffin-embedded colonic cancer tumour samples were sectioned $(4 \mu \mathrm{m})$, deparaffinized with xylene, rehydrated using a reduced ethanol gradient and then washed with deionized water. Antigen retrieval was performed in $10 \mathrm{mM}$ citrate buffer ( $\mathrm{pH}$ 6.0, S1699; Dako, Glostrup, Denmark) using a pressure cooker. Washing buffer consisted of $50 \mathrm{mM}$ Tris, $0.15 \mathrm{M}$ $\mathrm{NaCl}, \mathrm{pH} 7.6$, and $0.05 \%$ Tween-20. Endogenous peroxidase activity was first blocked with a dual endogenous enzyme block (Dako) for 10-20 min and then with a specific serum-free protein block (Dako) for $30 \mathrm{~min}$. Each primary antibody was diluted with an antibody diluent (Dako). The following antibodies were used: mouse monoclonal anti-ALDH1 (1:1400; clone: 44/ALDH; BD Biosciences, San Jose, CA, USA), mouse monoclonal anti-Achaete Scute homolog 2 (ASCL2; clone 7E2, 1:200 or 1:750; Merck Millipore, Burlington, MA, USA), rabbit monoclonal antibody to double cortin-like kinase 1 (DCLK1; clone EPR6085, 1:600 or 1:800; Abcam, Cambridge, UK), rabbit anti-15-PGDH (1:500; Novus Biologicals, Denver, CO, USA), rabbit anti-COX2 (1:400; Abcam, Cambridge, UK), and rabbit monoclonal anti-non-phospho (active) $\beta$-catenin (Ser45) (D2U8Y) (1:500; Cell Signaling, Leiden, the Netherlands). All incubations with a primary antibody were performed overnight in a humidity chamber at $4^{\circ} \mathrm{C}$. After washing, the sectioned tumour samples were incubated for $30 \mathrm{~min}$ at room temperature with a secondary antibody and developed using either an EnVision+ System-HRP labelled polymer anti-mouse or anti-rabbit antibody (K4000 and K4002 respectively; Dako), visualized by addition of 3,3'-diaminobenzidine (DAB) 


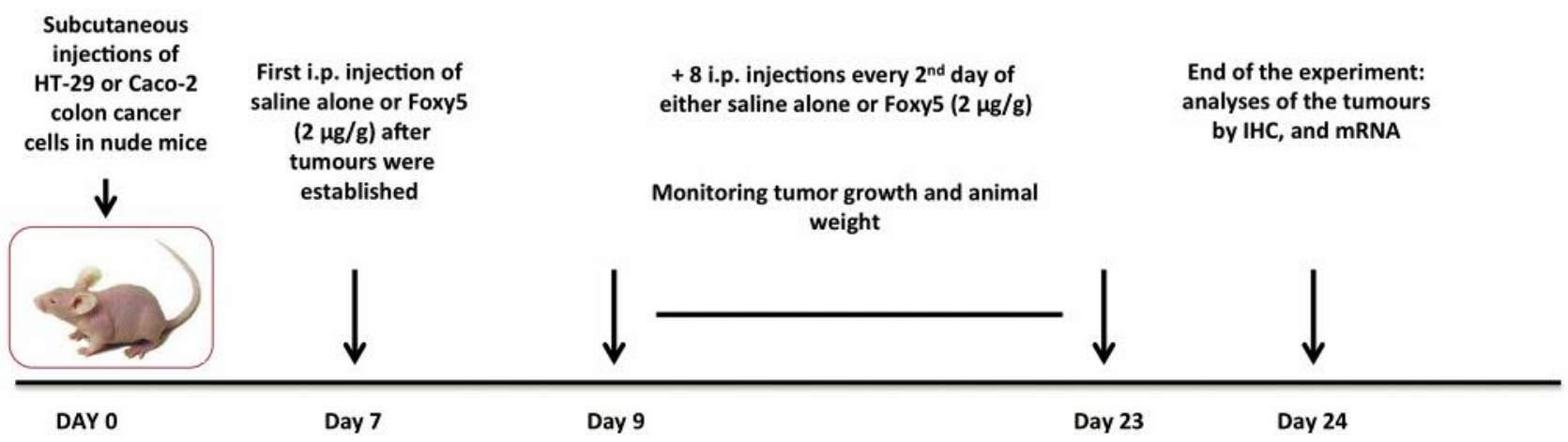

Figure 1. Schematic overview of the animal study design. IHC: Immunohistochemistry; i.p.: intraperitoneal.

substrate (Vector Laboratories Inc., Burlingame, CA, USA) for 2-5 min and then finally counterstained with haematoxylin.

The stained slides were scanned with a ScanScope CS (Aperio Technologies, Vista, CA, USA). The expression intensity levels of staining with each antibody, except for the non-phospho active $\beta$ catenin antibody, were semi-quantitatively evaluated using a visual grading system based on the overall staining intensity and percentage of positively stained cells. We placed six boxes $\left(0.3 \mathrm{~mm}^{2}\right.$ each, on an image at $20 \times$ magnification) over tumour cell growing areas and defined the staining as follows: $<5 \%$ positive cells was scored as $0, \leq 25 \%$ positive cells was scored as $1,26 \%$ to $50 \%$ positive cells was scored as $2,51 \%$ to $75 \%$ positive cells was scored as 3 , and staining of $>75 \%$ of cells was scored as 4 . The intensity of the stained tumour cells was graded as follows: No staining was scored as 0 , weak staining as 1 , moderate staining as 2 , and strong staining was scored as 3. A total staining score of between 0-12 was obtained for each sample by multiplying the score of the percentage of positively stained cells by the score of the intensity of positively stained cells. The mean of the scores of the six boxes constituted the score for each sample.

Staining with the non-phospho active $\beta$-catenin antibody was evaluated based on positive nuclear staining. For each section image, we placed six boxes $\left(0.08 \mathrm{~mm}^{2}\right.$ each, on an image at $40 \times$ magnification) over the tumour cell growing area. The number of cells positive for nuclear staining was defined for each box, and the average of the six boxes defined the non-phospho active $\beta$-catenin staining for each sample.

Real-time quantitative polymerase chain reaction $(q P C R)$. Total RNA from the tumour tissue samples was isolated using Qiagen RNeasy Plus Mini Kits (Qiagen GmbH, Hilden, Germany). The cDNA synthesis was performed using RevertAid H Minus M-MuLV reverse transcriptase (Thermo Fisher Scientific, Waltham, MA, USA). The following primers (from Applied Biosystems, Cambridge, UK) were used: ALDH1Al (Hs00946916_m1) and DCLK1 (Hs00178027_ml). Amplifications were performed in an Mx3005P system (Agilent Technologies, Inc., Santa Clara, CA, USA). The reactions were normalized to expression of the housekeeping gene hypoxanthine phosphoribosyltransferase 1 (HPRT1) (Hs99999909_m1) and analysed with MxPro qPCR software (Agilent Technologies). The tumour tissue data were expressed in relation to that detected in the respective cell line from which the tumour was derived.
Statistical analysis. Prism software 5.0 (GraphPad software, San Diego, CA, USA) was used for statistical analyses. Unpaired Student's $t$-test was used, and statistical significance was considered when the $p$-value was less than 0.05 .

\section{Results}

Study design. The animal experiment was designed so that Foxy5 peptide treatment began when a palpable xenograft tumour in the animals was present (Figure 1). We believe that this better reflects the clinical situation, where a patient always has a tumour detected before any type of treatment is initiated. We have previously used this approach with good results in an orthotopic metastatic mouse model (15).

Foxy5 reduces the expression of $A L D H$ and DCLK1 in colonic tumours. The results outlined in Figure 2 show the in vivo effect of Foxy5 treatment on expression of ALDH protein in HT-29 and Caco-2 colonic cancer xenograft tissue as visualized by IHC. Representative images from vehicleand Foxy5-treated animals are presented for both cell lines (Figure 2A and B). The results clearly show that treatment with the Foxy5 peptide reduced the IHC staining for ALDH in both cell lines (Figure 2A and B). Although ALDH is a general stem cell marker, we believe that in this case, it reflects the staining of colonic CSCs, as the analyses were performed on identified colonic cancer tissues (17). However, we further confirmed this assumption by staining the tumour samples with the colon CSC marker DCLK1 (17-19).

Using the same tumours and evaluation approach, we next analysed the IHC staining of the colon-cancer-specific stem cell marker DCLK1 following treatment with either vehicle alone or with Foxy5 in HT-29 and Caco-2 cell-derived xenograft tissues. We obtained nearly identical results with the antibody for DCLK1 as with that for ALDH, thus demonstrating that Foxy5 treatment significantly reduced the IHC staining of colonic CSCs in both HT-29 and Caco-2 cancer tissue (Figure 2C and D). 


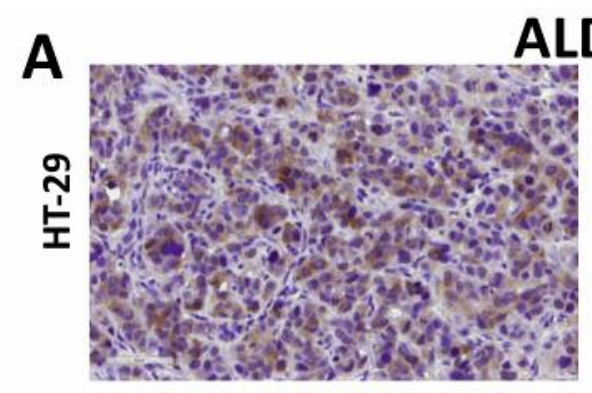

\section{ALDH}
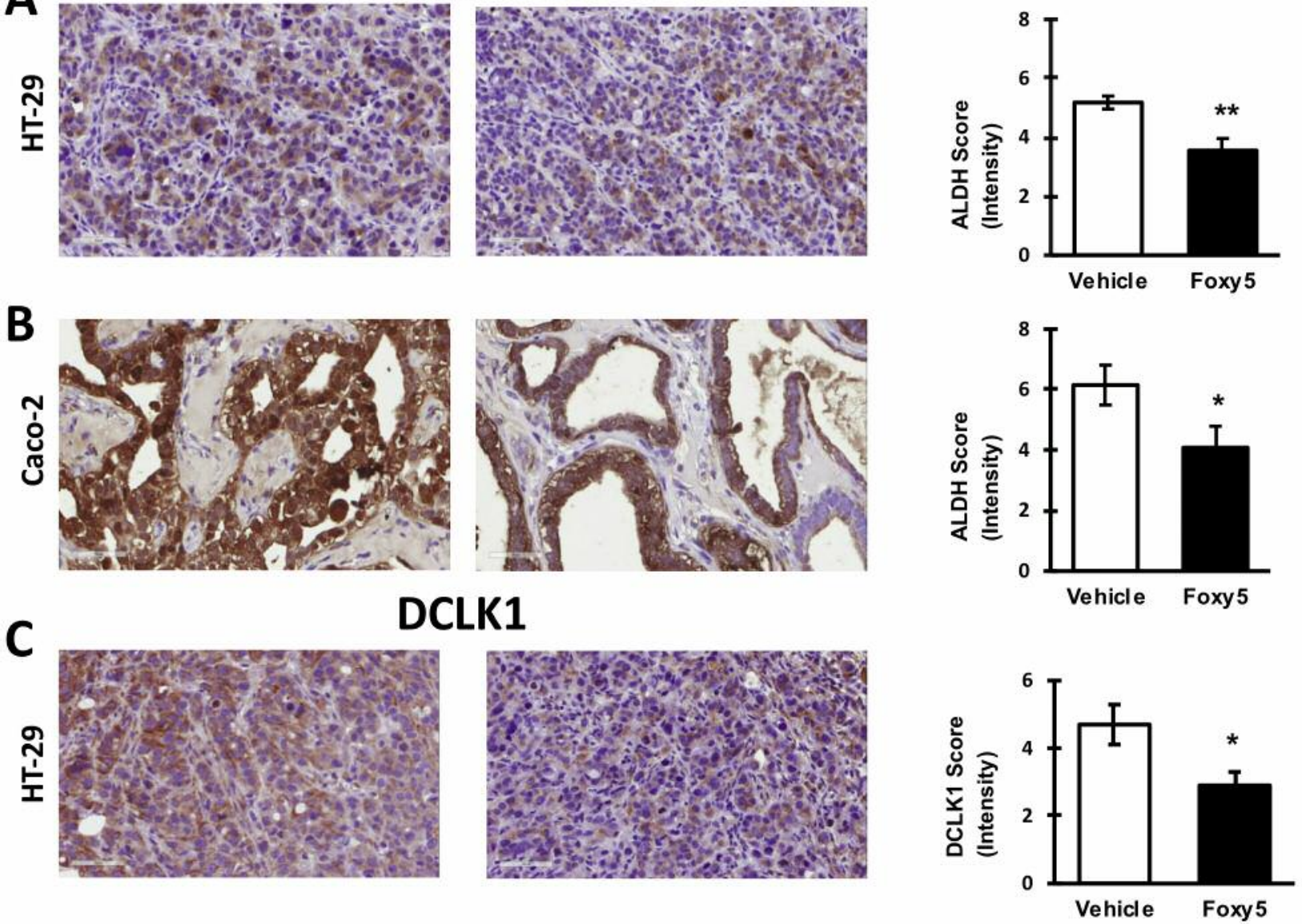

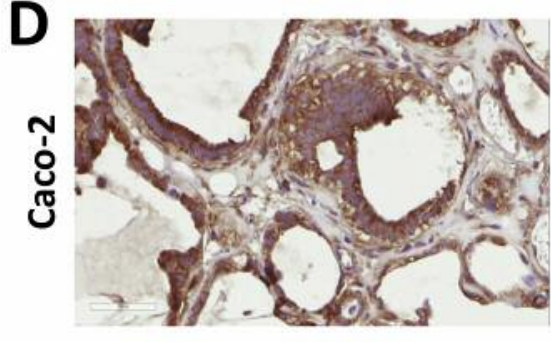

Vehicle

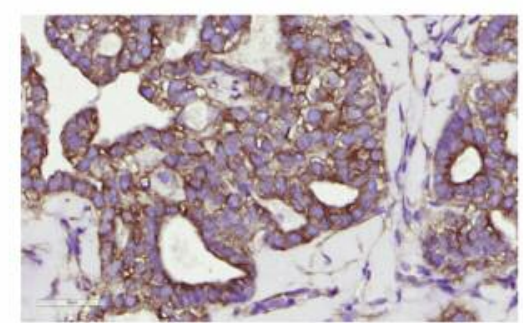

Foxy5

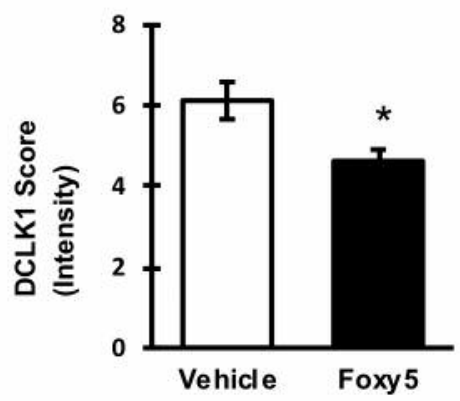

Figure 2. In vivo effect of Foxy5 treatment on aldehyde dehydrogenase (ALDH; A and B) and double cortin-like kinase 1 (DCLK1; C and D) protein expression in HT-29 and Caco-2 cell-derived xenograft tissue as visualized by immunohistochemistry. Representative images from vehicle- and Foxy5treated animals are presented. For each slide, six boxes were randomly placed over the images of stained tumour tissues; for each box, staining was scored as the percentage of stained cells multiplied by the staining intensity (see the Materials and Methods section). The mean value was obtained from the six boxes for each tumour sample. The intensity scores from all included data are given as the mean \pm SEM. Significantly different at: *p<0.05 and $* * p<0.01$.

These data were further validated by real-time qPCR in Caco-2-derived tumours that were immediately frozen for subsequent mRNA analyses upon termination of the animal experiments. The results from these analyses confirmed that treatment with Foxy5 significantly reduced the mRNA expression of both $A L D H$ and $D C L K 1$ in tumour tissues (Figure 3).

Foxy5-induced effects on $P G E_{2}$-generating and -degrading enzymes. The lipid mediator $\mathrm{PGE}_{2}$ has been demonstrated 

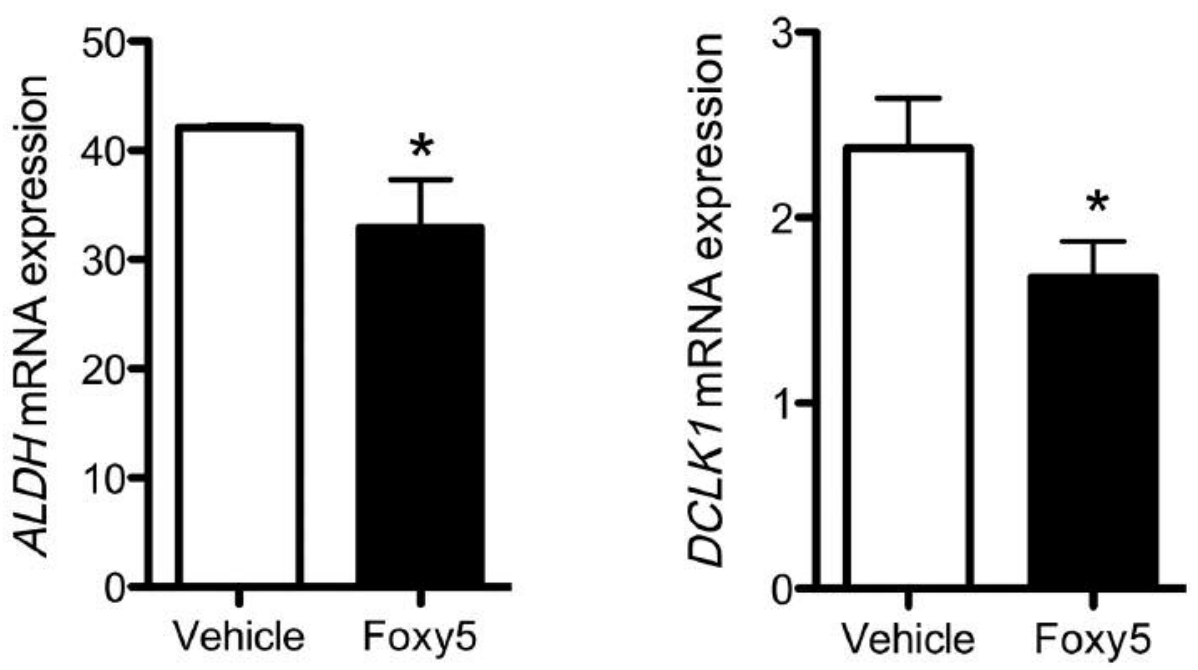

Figure 3. In vivo effects of Foxy5 treatment on aldehyde dehydrogenase (ALDH) and double cortin-like kinase 1 (DCLK1) mRNA expression in Caco-2 cell-derived xenograft tissue. The results of Foxy 5 treatment were compared with those of tumours from vehicle-treated control mice. The $m R N A$ results from all included mice are given as the mean $\pm S E M$. *Significantly different at $p<0.05$.

to positively regulate the colonic CSC niche (6). Therefore, we analysed the effect of Foxy5 treatment on the protein expression of the $\mathrm{PGE}_{2}$-generating enzyme COX2 in HT-29 and Caco-2 cell-derived xenograft tissues by IHC. Representative images from vehicle- and Foxy5treated animals are presented, and they show, as do the intensity scores, that the WNT5A-derived peptide statistically significantly reduced the level of COX2 in both HT-29 and Caco-2 cell-derived xenograft tumours (Figure 4A and B).

In addition to the analysis of the $\mathrm{PGE}_{2}$-generating enzyme $\mathrm{COX} 2$, we also analysed the expression of the $\mathrm{PGE}_{2}-$ degrading enzyme 15-PGDH. Figure $4 \mathrm{C}$ and $\mathrm{D}$ shows the in vivo effect of Foxy5 treatment on 15-PGDH protein expression in both HT-29 and Caco- 2 cell-derived xenograft tissue, as visualized by IHC. Representative images from vehicle- and Foxy5-treated animals are presented together with the intensity scores from all included data from both HT-29 and Caco-2 cell-derived xenograft tumours. In contrast to reducing COX2 protein expression, treatment with Foxy5 significantly increased the expression of 15PGDH in these tumour tissues (Figure $4 \mathrm{C}$ and D). These opposite effects on COX2 and 15-PGDH expression indicate a reduced activity of the $\mathrm{PGE}_{2}$ pathway, in good agreement with our observation of a Foxy5-mediated reduction of colonic CSCs in these tumours.

Foxy5 impairs $\beta$-catenin signalling in colonic cancer tumours. For many years, $\beta$-catenin signalling has been documented as an important regulator of intestinal stem cells (20) and also of colonic CSCs (21). Therefore, we analyzed the in vivo effects of Foxy5 treatment on $\beta$ catenin signalling in both HT-29- and Caco-2 cell-derived colonic cancer xenograft tissue. It is well established that the unphosphorylated form of $\beta$-catenin is the active form of this protein that goes into the cell nuclei, where it triggers transcription of its different downstream targets (20-22). Consequently, we first analysed the expression of active $\beta$-catenin in the nuclei of the tumour tissues from animals treated with vehicle alone or with Foxy5. Representative images from both HT-29 and Caco- 2 cellderived tumours as well as the scores from these data are outlined in Figure 5A and B. The results clearly show that Foxy 5 treatment caused significant reduction in $\beta$-catenin signalling.

To further validate these findings, we also analysed the expression of ASCL2 protein in these tumours. ASCL2 is a transcription factor activated by $\beta$-catenin signalling that has been shown to promote the CSC niche (23). Representative images of ASCL2 staining of HT-29 and Caco-2 cell-derived tumours from vehicle- and Foxy5-treated animals are presented together with the ASCL2 staining scores (Figure $5 \mathrm{C}$ and D). In accordance with the ability of Foxy5 to reduce $\beta$-catenin signalling (Figure 5A and B), we also observed similar Foxy5-induced reduction of the expression of the downstream $\beta$-catenin target ASCL2 (Figure 5C and D).

Finally, we validated the ability of Foxy5 to reduce $\beta$ catenin signalling by estimating the tumour volumes in animals treated with vehicle alone or with Foxy5. The results from HT-29- and Caco-2-derived tumours clearly show that Foxy5 impaired the growth of both types of tumours (Figure 6). 

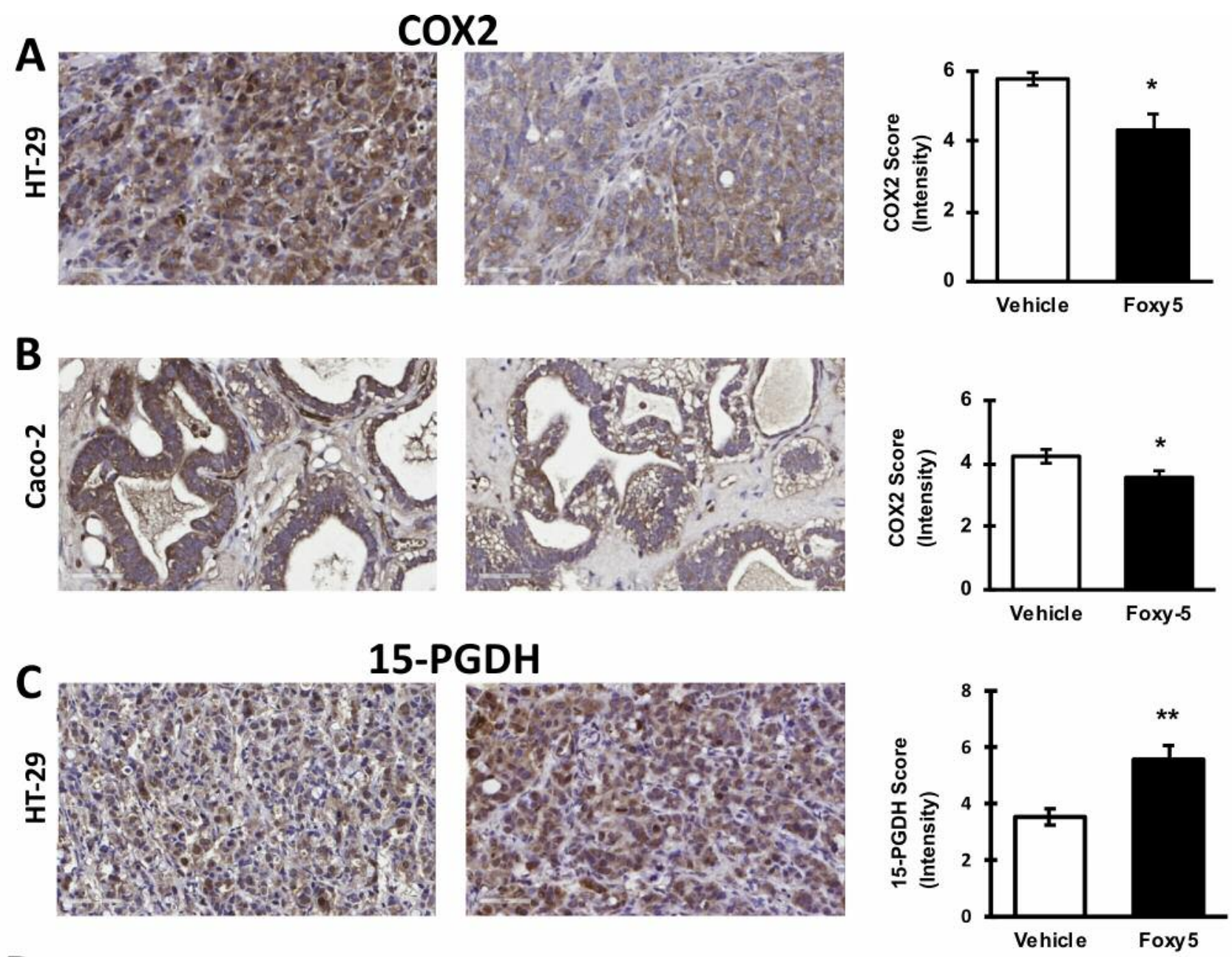

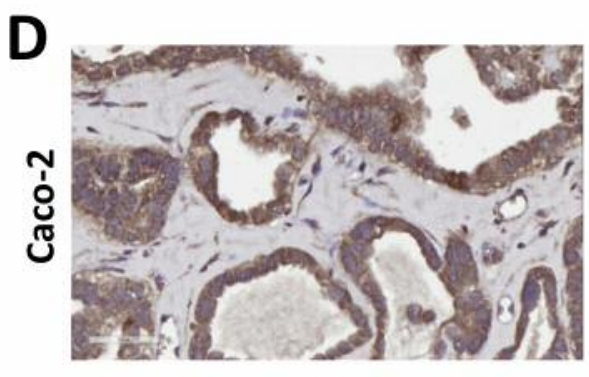

Vehicle

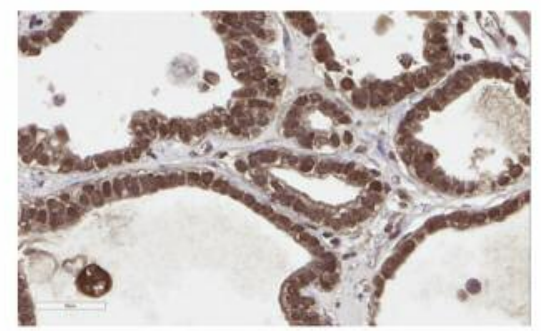

Foxy5

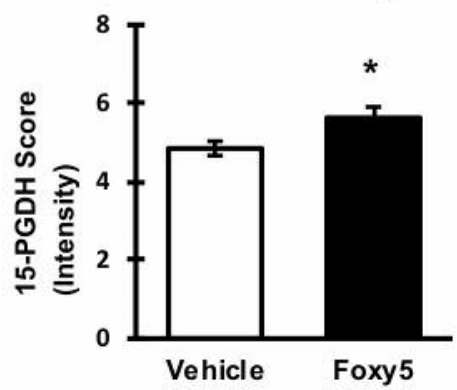

Figure 4. In vivo effect of Foxy5 treatment on COX2 (A and B) and 15-PGDH (C and D) protein expression in HT-29 and Caco-2 cell-derived xenograft tissue as visualized by immunohistochemistry. Representative images from vehicle- and Foxy5-treated animals are presented. For each slide, six boxes were randomly placed over the images of stained tumour tissues; for each box, staining was scored as the percentage of stained cells multiplied by the staining intensity as described in the Materials and Methods section. The intensity scores from all included data are given as the mean \pm SEM. Significantly different at $* p<0.05 ; * * p<0.01$.

\section{Discussion}

CSCs are disseminated into the primary tumour mass and can also spread to other sites in the body, where they can remain dormant for years. At such sites, CSCs interact with the tumour microenvironment, which is essential for the functional behaviour of CSCs (24) and makes it possible for CSCs to maintain their stemness properties. This is the most 

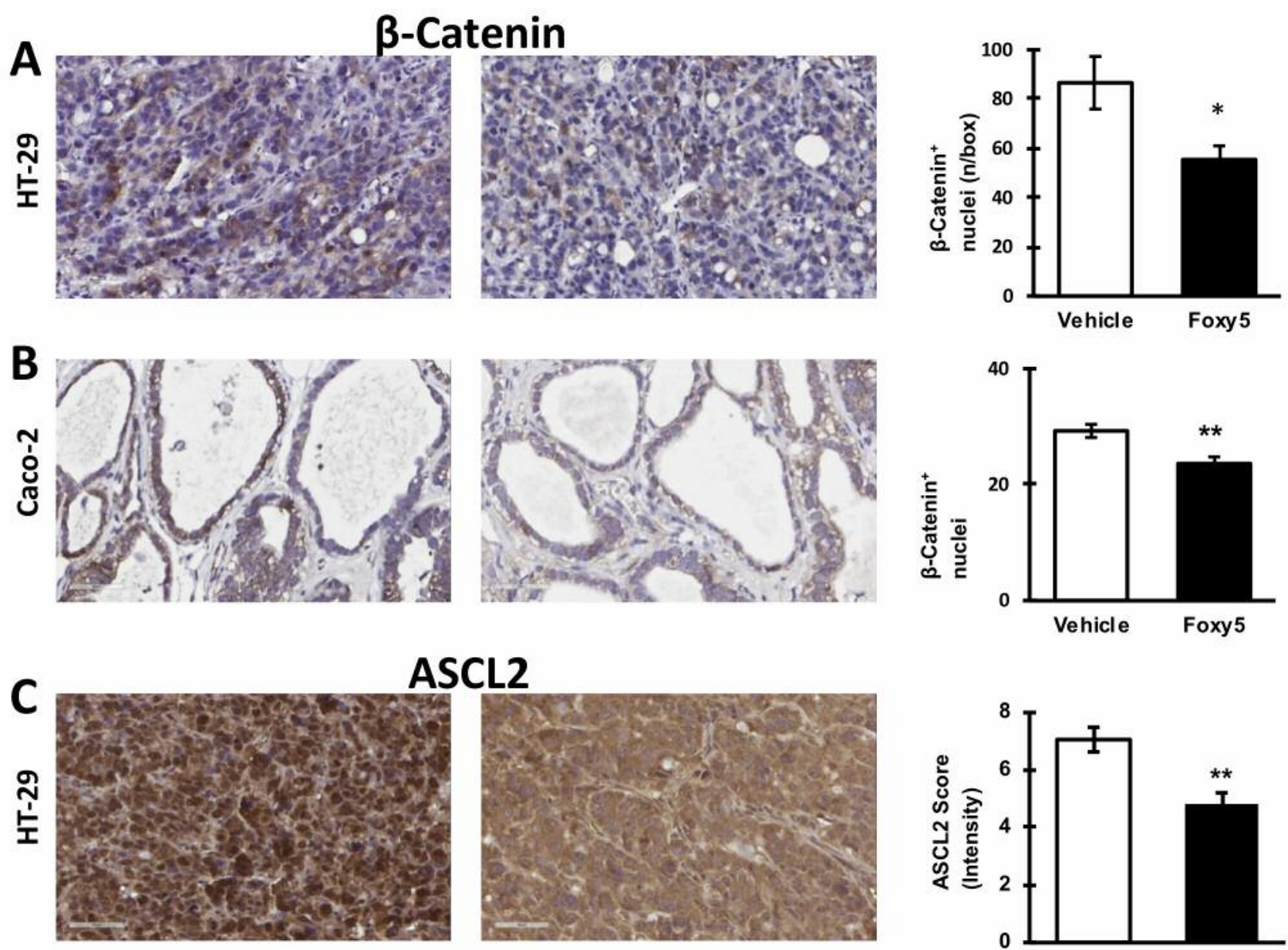

ASCL2

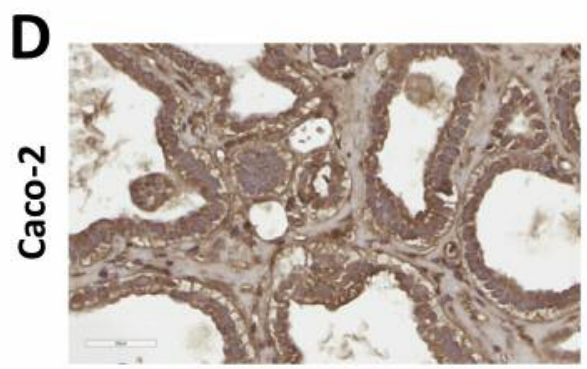

Vehicle
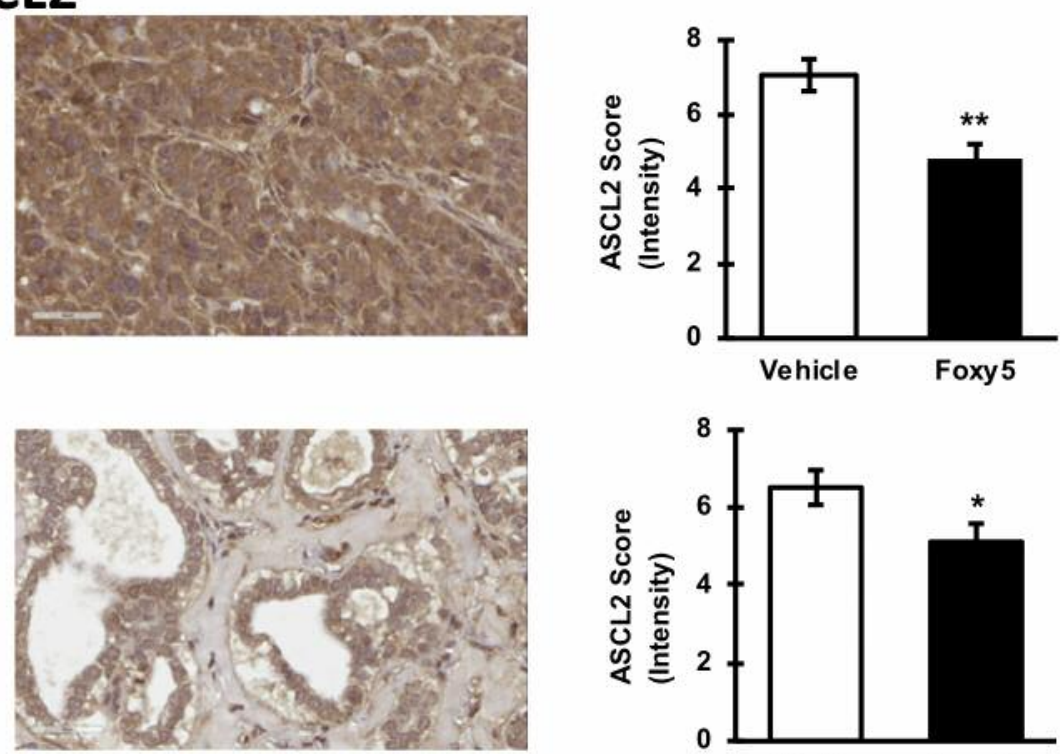

Foxy5

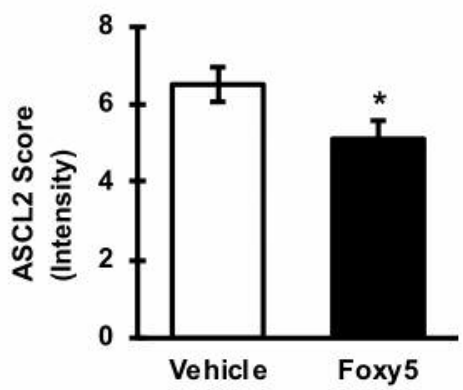

Figure 5. In vivo effect of Foxy5 treatment on nuclear active $\beta$-catenin ( $A$ and B) and ASCL2 (C and D) protein expression in HT-29 and Caco-2 cell-derived xenograft tissue, as visualized by immunohistochemistry. Representative images from vehicle-and Foxy5-treated animals are presented. For each slide, six boxes were randomly placed over the images of stained tumour tissues and evaluated as described in the Materials and Methods section. The scores from all included data are given as the mean \pm SEM. Significantly different at $* p<0.05$ and $* * p<0.01$.

probable reason why it is so difficult to study the regulation of CSCs in vitro. Based on these findings, studies and evaluation of possible target molecules that can be used in preventing the maintenance or even expansion of CSCs are most adequately investigated in an in vivo setting.
In our initial analyses of the number of CSCs in tumour tissue, we employed an antibody to ALDH which also recognizes normal stem cells. However, because we only analysed ALDH staining in morphologically defined tumour areas, we believed that such staining most probably 
HT-29 cells

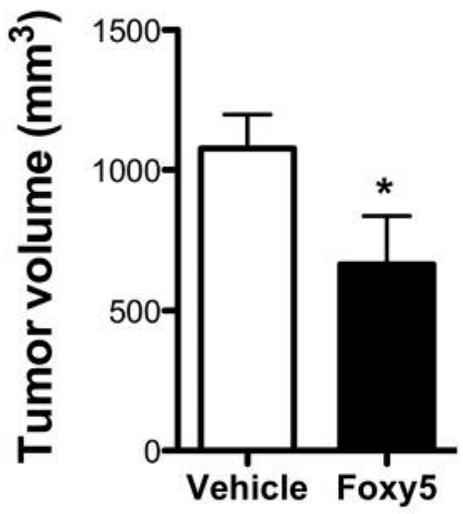

Caco-2 cells

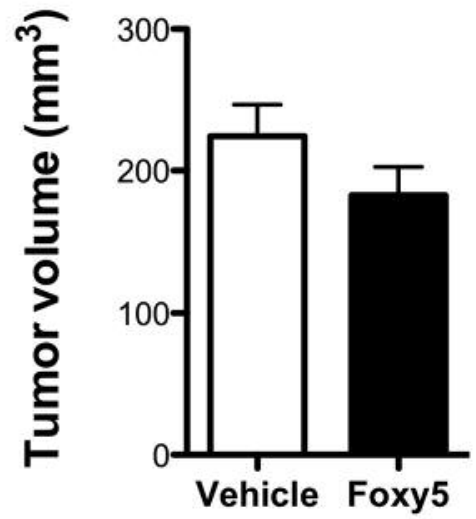

Figure 6. Effect of Foxy5 on tumour volumes of HT-29- and Caco-2 cell-derived xenografts. All tumour diameters (d1 and d2) were determined with a calliper the day before the animals were sacrificed, and the volume of each tumour was then calculated according to the formula, $V=(\pi / 6)\left(d_{1} \times d_{2}\right)^{3 / 2}(16)$. The results from HT-29- and Caco-2-derived tumours are given as the mean \pm SEM. *Significantly different at $p<0.05$.

represented the number of CSCs. To validate this assumption, we also stained these colon tumour tissues with an antibody to DCLK1, as DCLK1 has been shown to be a specific marker of colonic CSCs $(18,19)$. More recently, DCLK1 was also documented to possess regulatory functions in colonic CSCs (19).

Although we cannot state the mechanism(s) behind the ability of the Foxy5 peptide to reduce the number of colonic CSCs in the present study, we do not believe that it directly kills the colonic CSCs. Instead, we believe that the Foxy5 peptide impairs the ability of these cells to maintain their stemness properties. This would lead to the differentiation of these CSCs into colonic cancer cells without stem cell characteristics. This assumption means that if a patient with colonic cancer were to be treated with a combination, sequentially or simultaneously, of Foxy 5 and chemotherapy, the effect of Foxy5 of reducing the number of CSCs and making them differentiate into colonic cancer cells without stem cell properties would logically also make them sensitive to chemotherapeutic agents given to the patient. Overall, this would have the potential not only to significantly improve the effect of chemotherapy but also to significantly reduce the risk of cancer recurrence.

To further validate the present findings revealing a reduction in the number of colonic CSCs in an in vivo setting, we explored whether Foxy5 treatment could impair intracellular signals known to be up-regulated in colonic cancer tissue and previously implicated in the promotion of colonic CSCs. In good agreement with our previous in vitro data (7), Foxy5 treatment of mice bearing either HT-29 or Caco- 2 cell-derived tumours also reduced $\beta$-catenin signalling in vivo. Foxy5 may, therefore, influence the number of colonic CSCs by a similar mechanism as any of the WNT/ $\beta$-catenin inhibitors presently investigated in different clinical trials. However, $\beta$-catenin signalling is not the only mechanism demonstrated to promote colonic CSCs. It has also been shown that $\mathrm{PGE}_{2}$ signalling promotes the niche of colonic CSCs and, creating a tumor microenvironment enriched in myeloid-derived suppressor cells $(6,24)$. The basis for increased $\mathrm{PGE}_{2}$ signalling in colonic cancer tissue is directly related to the up-regulation of the $\mathrm{PGE}_{2}$ generating enzyme COX2 and down-regulation of the $\mathrm{PGE}_{2}$-degrading enzyme 15-PGDH (25). The present in vivo findings that Foxy5 treatment of colonic cancer derived from two different human colonic cancer cell lines reduced the expression of COX 2 and simultaneously increased the expression of 15-PGDH reveal that Foxy 5 can target three different targets ( $\beta$-catenin signalling, $\mathrm{COX} 2$ and 15-PGDH) and thereby effectively impair the number of colonic CSCs.

Taken together, these novel data suggest that Foxy5 has a broad and general tumour-suppressive effect on colonic cancer cells. These effects include impaired invasive migration (7), but also reduced tumour growth and, most importantly, a reduction in the number of colonic CSCs. The latter finding provides the basis for a novel therapeutic strategy with regard to how Foxy5 treatment can be further optimized in the future treatment of patients with colonic cancer.

\section{Conflicts of Interest}

T.A. is a shareholder of WntResearch $\mathrm{AB}$ and is the part time Chief Scientific Officer of WntResearch AB. This does not alter the Author's adherence to all guidelines for publication in Anticancer Research. 


\section{Authors' Contributions}

AS initiated and designed the study. TA co-designed the study. AS and TA evaluated the IHC results and wrote the article. JO and $\mathrm{KB}$ performed the animal experiments and QL performed the IHC work and evaluated the results. All Authors read and approved the final article.

\section{Acknowledgements}

Research Grants from the Royal Physiographic Society in Lund (to J.O., K.B.), the Swedish Cancer Foundation, the Swedish Research Council, the UMAS Cancer Foundation and WntResearch AB supported Dr. Sjölander's research group. The Swedish Cancer Foundation, the Swedish Research Council, the Skåne University Hospital Research Foundation, and the UMAS Cancer Foundation supported Dr. Andersson's research group. The Authors thank Gunilla Jönsson for her technical assistance. The founders had no role in the preparation of this study and writing of this article.

\section{References}

1 Society AC: Colorectal Cancer Facts \& Figures 2017-2019. Atlanta: American Cancer Society 2017.

2 Sargent DJ, Wieand HS, Haller DG, Gray R, Benedetti JK, Buyse M, Labianca R, Seitz JF, O’Callaghan CJ, Francini G, Grothey A, O'Connell M, Catalano PJ, Blanke CD, Kerr D, Green E, Wolmark N, Andre T, Goldberg RM and De Gramont A: Disease-free survival versus overall survival as a primary end point for adjuvant colon cancer studies: Individual patient data from 20,898 patients on 18 randomized trials. J Clin Oncol 23: 8664-8670, 2005. PMID: 16260700. DOI: 10.1200/ JCO. 2005.01.6071

3 Giantonio BJ, Catalano PJ, Meropol, NJ, O’Dwyer PJ, Mitchell EP, Alberts SR, Schwartz MA and Benson AB III: Bevacizumab in combination with oxaliplatin, fluorouracil and leucovorin (FOLFOX4) for previously treated metastatic colorectal cancer: Results from the Eastern Cooperative Oncology Group Study E3200. J Clin Oncol 25: 1539-1544, 2007. PMID: 17442997. DOI: $10.1200 / J C O .2006 .09 .6305$

4 Siegel R, DeSantis C, Virgo K, Stein K, Mariotto, A, Smith T, Cooper D, Gansler T, Lerro C, Fedewa S, Lin C, Leach C, Cannady RS, Cho H, Scoppa S, Hachey M, Kirch R, Jemal A and Ward E: Cancer treatment and survivorship statistics, 2012. CA Cancer J Clin 62: 220-241, 2012. PMID: 22700443. DOI: 10.3322/caac.21149

5 Ricci-Vitiani L, Lombardi DG, Pilozzi E, Biffoni M, Todaro M, Peschle C and De Maria R: Identification and expansion of human colon-cancer-initiating cells, Nature 445: 111-115, 2007. PMID: 17122771. DOI: $10.1038 /$ nature05384

6 Wang D, Fu L, Sun H, Guo L and DuBois RN: Prostaglandin E promotes colorectal cancer stem cell expansion and metastasis in mice. Gastroenterolology 149: 1884-1895, 2015. PMID: 26261008. DOI: 10.1053/j.gastro.2015.07.064

7 Mehdawi ML, Prasad CP, Ehrnström R, Liu Q, Andersson T and Sjölander A: Non-canonical WNT5A signaling up-regulates the expression of the tumor suppressor 15-PGDH and induces differentiation of colon cancer cells. Molecular Oncology 10: 1415-1429, 2016. PMID: 27522468. DOI: 10.1016/j.molonc. 2016.07.011
8 Kumawat K and Gosens R: WNT-5A; signaling and functions in health and disease. Cell Mol Life Sci 73: 567-587, 2016. PMID: 26514730. DOI: 10.1007/s00018-015-2076-y

9 Prasad CP, Manchanda M, Mohapatra P and Andersson T: WNT5A as a therapeutic target in breast cancer. Cancer Metast Rev 37(4): 767-778, 2018. PMID: 30171384. DOI: 10.1007/ s10555-018-9760-y

10 Kikuchi A, Yamamoto H, Sato A and Matsumoto S: WNT5A: Its signalling, functions and implication in diseases. Acta Physiol 204: 17-33, 2012. PMID: 21518267. DOI: 10.1111/j.1748-1716. 2011.02294.x

11 Dejmek J, Dejmek A, Säfholm A, Sjölander A and Andersson T: WNT-5a protein expression in primary Dukes B colon cancers identifies a subgroup of patients with good prognosis. Cancer Res 65: 9142-9146, 2005. PMID:16230369. DOI: 10.1158/00085472.CAN-05-1710

12 Säfholm A, Leandersson K, Dejmek J, Nielsen CK, Villoutreix BO and Andersson T: A formylated hexapeptide ligand mimics the ability of WNT-5A to impair migration of human breast epithelial cells. J Biol Chem 281: 2740-2749, 2006. PMID: 16330545. DOI: $10.1074 /$ jbc.M508386200

13 Säfholm A, Tuomela J, Rosenkvist J, Dejmek J, Härkönen P and Andersson T: The WNT-5A-derived hexapeptide Foxy-5 inhibits breast cancer metastasis in vivo by targeting cell motility. Clin Cancer Res 14: 6556-6563, 2008. PMID: 18927296. DOI: 10.1158/1078-0432.CCR-08-0711

14 Savari S, Liu M, Zhang Y, Sime W and Sjölander A: CysLT(1)R antagonists inhibit tumor growth in a xenograft model of colon cancer. PloS One 8: e73466, 2013. PMID: 24039952. DOI: 10.1371/journal.pone.0073466

15 Canesin G, Evans-Axelsson S, Hellsten R, Krzyzanowska A, Prasad CP, Bjartell A and Andersson T: Treatment with the WNT5A-mimicking peptide Foxy-5 effectively reduces the metastatic spread of WNT5A-low prostate cancer cells in an orthotopic mouse model. PLoS One 12: e0184418, 2017. PMID: 28886116. DOI: 10.1371/journal.pone .0184418

16 Feldman JP, Goldwasser R, Mark S, Schwartz J and Orion I: A mathematical model for tumor volume evaluation using two dimensions. J Appl Quant Methods 4: 455-462, 2009.

17 Bellamkonda K, Satapathy SR, Douglas D, Chandrashekar N, Selvanesan BC, Liu M, Savari S, Jönsson G and Sjölander A: Montelukast, a CysLT1 receptor antagonist, reduces colon cancer stemness and tumor burden in a mouse xenograft model of human colon cancer. Cancer Lett 437: 13-24, 2018. PMID: 30144515. DOI: 10.1016/j.canlet.2018.08.019

18 Nakanishi Y, Seno H, Fukuoka A, Ueo T, Yamaga Y, Maruno T, Nakanishi N, Kanda K, Komekado H, Kawada M, Isomura A, Kawada K, Sakai Y, Yanagita M, Kageyama R, Kawaguchi Y, Taketo MM, Yonehara S and Chiba T: DCLK1 distinguishes between tumor and normal stem cells in the intestine. Nature Genet 45: 98-103, 2013. PMID: 23202126. DOI: $10.1038 / \mathrm{ng} .2481$

19 Sarkar S, Popov VL, O’Connell MR, Stevenson HL, Lee BS, Obeid RA, Luthra GK and Singh P: A novel antibody against cancer stem cell biomarker, DCLK1-S, is potentially useful for assessing colon cancer risk after screening colonoscopy. Lab Invest 97: 1245-1261, 2017. PMID: 28414327. DOI: 10.1038/ labinvest.2017.40

20 van der Flier LG and Clevers H: Stem cells, self-renewal and differentiation in the intestinal epithelium. Annu Rev Physiol 71: 241-260, 2009. PMID: 18808327. DOI: 10.1146/annurev. physiol.010908.163145 
21 Hua F, Shang S, Yang YW, Zhang HZ, Xu TL, Yu JJ, Zhou DD, Cui B, Li K, Lv XX, Zhang XW, Liu SS, Yu JM, Wang F, Zhang $\mathrm{C}$, Huang $\mathrm{B}$ and $\mathrm{Hu} \mathrm{ZW}$ : TRIB3 interacts with $\beta$-catenin and TCF4 to increase stem cell features of colorectal cancer stem cells and tumorigenesis. Gastroenterology 156(3): 708-721, 2019. PMID: 30365932. DOI: 10.1053/j.gastro.2018.10.031

22 Salim T, Sjölander A. and Sand-Dejmek J: Nuclear expression of glycogen synthase kinase-3beta and lack of membranous betacatenin is correlated with poor survival in colon cancer. Int $\mathbf{J}$ Cancer 133: 807-815, 2013. PMID: 23389968. DOI: 10.1002/ ijc. 28074

23 Schuijers J, Junker JP, Mokry M, Hatzis P, Koo BK, Sasselli V, van der Flier LG, Cuppen E, van Oudenaarden A and Clevers H: ASCL2 acts as an R-spondin/Wnt-responsive switch to control stemness in intestinal crypts. Cell Stem Cell 16(2): 158-170, 2015. PMID: 25620640. DOI: 10.1016/j.stem.2014.12.006
24 Sica A, Porta C, Amadori A and Pastò A: Tumor-associated myelod cells as guiding forces for cancer cell stemness. Cancer Immunol Immunother 66: 1025-1036, 2017. PMID 28401258. DOI: $10.1007 / \mathrm{s} 00262-017-1997-8$

25 Backlund MG, Mann JR, Holla VR, Buchanan FG, Tai HH, Musiek ES, Milne GL, Katkuri S and DuBois RN: 15Hydroxyprostaglandin dehydrogenase is down-regulated in colorectal cancer. J Biol Chem 280: 3217-3223, 2005. PMID: 15542609. DOI: $10.1074 /$ jbc.M411221200

Received February 10, 2019

Revised March 12, 2019

Accepted March 20, 2019 